DOI: $10.1515 /$ awutm -2015-0008

$\frac{\mathrm{DE}}{\mathrm{G}}$
Analele Universităţii de Vest,

Timişoara

Seria Matematică - Informatică

LIII, 1, (2015), 151- 161

\title{
Strong convergence of the Ishikawa iteration for Lipschitz $\alpha$-hemicontractive mappings
}

Micah Okwuchukwu Osilike and Anthony Chibuike Onah

\begin{abstract}
A new class of $\alpha$-hemicontractive maps $T$ for which the strong convergence of the Ishikawa iteration algorithm to a fixed point of $T$ is assured is introduced and studied. The study is a continuation of a recent study of a new class of $\alpha$-demicontractive mappings $T$ by L. Măruşter and Ș. Măruşter, Mathematical and Computer Modeling 54 (2011) 2486-2492 in which they proved strong convergence of the Mann iteration scheme to a fixed point of $T$. Our class of $\alpha$-hemicontractive maps is more general than the class of $\alpha$-demicontractive maps. No compactness assumption is imposed on the operator or it's domain, and no additional requirement is imposed on the set of fixed points.
\end{abstract}

AMS Subject Classification (2000). 65J15

Keywords. $\alpha$-demicontractive maps, $\alpha$-hemicontractive maps, fixed points, Ishikawa iteration, strong convergence.

\section{Introduction}

Let $H$ be a real Hilbert space with inner product $\langle.,$.$\rangle and induced norm \|$.$\| .$ Let $C$ be a nonempty closed convex subset of $\mathrm{H}$.

Definition 1.1. A mapping $T: C \rightarrow C$ is said to be demicontractive (see for example [1]) if $F(T):=\{x \in C: T x=x\} \neq \emptyset$ and there exists $k \in[0,1)$ 
such that

$$
\|T x-p\|^{2} \leq\|x-p\|^{2}+k\|x-T x\|^{2}, \forall x \in C \text { and } \forall p \in F(T) .
$$

The class of demicontractive maps coincides with the class of mappings satisfying condition (A) which was studied by Ş. Măruşter $[2,3]$.

Definition 1.2. $T: C \rightarrow C$ is said to satisfy condition (A) if $F(T) \neq \emptyset$ and there exists $\lambda>0$ such that

$$
\langle x-T x, x-p\rangle \geq \lambda\|x-T x\|^{2}, \forall x \in C \text { and } \forall p \in F(T) .
$$

Definition 1.3. $T$ is said to be hemicontractive (see for example [4]) if $k=1$ in (1.1).

The class of demicontractive maps is a proper subclass of the class of hemicontractive maps (see for example [4]). The classes of demicontractive maps and hemicontractive maps have been studied by many authors (see for example [1-14]).

The Mann iteration scheme $\left\{x_{n}\right\}_{n=1}^{\infty}$ generated from an arbitrary $x_{1} \in C$ by

$$
x_{n+1}=\left(1-\alpha_{n}\right) x_{n}+\alpha_{n} T x_{n}, n \geq 1,
$$

where the control sequence $\left\{\alpha_{n}\right\}_{n=1}^{\infty}$ is a real sequence in $(0,1]$ satisfying some appropriate conditions has been used by several authors for the approximation of fixed points of demicontractive maps. It is now well known (see for example [15]) that Mann iteration scheme may not in general converge to a fixed point of a hemicontractive map in Hilbert spaces. For hemicontractive maps, the Ishikawa iteration sequence $\left\{x_{n}\right\}_{n=1}^{\infty}$ generated from arbitrary $x_{1} \in C$ by

$$
x_{n+1}=\left(1-\alpha_{n}\right) x_{n}+\alpha_{n} T\left[\left(1-\beta_{n}\right) x_{n}+\beta_{n} T x_{n}\right], n \geq 1,
$$

where $\left\{\alpha_{n}\right\}$ and $\left\{\beta_{n}\right\}$ are control sequences in $[0,1]$ is usually applicable.

Demicontractivity of $T$ alone is not sufficient for the convergence of the Mann iteration to a fixed point of $T$ even in finite dimensional spaces (see for example [17]). Some additional smoothness properties of $T$ are necessary, like continuity or demiclosedness principle.

Definition 1.4. $T: C \rightarrow H$ is said to be demiclosed at $p$ if whenever $\left\{x_{n}\right\}_{n=1}^{\infty}$ is a sequence in $C$ which converges weakly to $x^{*}$ in $C$ and $\left\{T x_{n}\right\}_{n=1}^{\infty}$ converges strongly to $p$, then $T x^{*}=p$.

In finite dimensional spaces, Măruşter proved the following: 
Theorem 1.1. ([2]) Let $\Re^{m}$ be the Euclidean m-dimensional space and let $T: \Re^{m} \rightarrow \Re^{m}$ be a nonlinear mapping satisfying the conditions:

(i) $(I-T)$ is demiclosed at 0 ;

(ii) $T$ is demicontractive with constant $k$, or, equivalently $T$ satisfies condition (A) with $\lambda=\frac{(1-k)}{2}$;

(iii) $0<a<\alpha_{n} \leq b<2 \lambda=1-k$. Then the Mann iteration sequence $\left\{x_{n}\right\}$ converges to a point of $F(T)$ for any starting point $x_{0} \in \Re^{m}$.

In infinite dimensional spaces, the two conditions (demicontractivity and demiclosedness principle) are not sufficient for strong convergence (see for example $[3,17])$. The two conditions however ensure weak convergence of $\left\{x_{n}\right\}$ to a fixed point of $T$ in real Hilbert spaces and some more general Banach spaces (see for example $[1,3,8]$ ). In order to obtain strong convergence, some additional conditions or some modifications of the standard Mann iteration are necessary. Such modifications have been considered by several authors (see for example $[3,5,6,8,10-12,18-20]$ ).

In [3] the existence of a nonzero solution $h \in H, h \neq 0$, of the variational inequality

$$
\langle x-T x, h\rangle \leq 0, \forall x \in H
$$

is required as an additional condition for strong convergence. The results of [3] has been extended by some authors to either more general Banach spaces or to the Ishikawa iteration scheme (see for example $[8,11-13]$ ). We note however that the existence of a nonzero solution of the variational inequality (1.5) exists only in very particular cases.

In exploring more conditions that may be less restrictive than the condition of the existence of a nonzero solution of (1.5), L. Măruşter and S. Măruşter [17] introduced a new concept of demicontractivity called $\alpha$-demicontractivity.

Definition 1.5. A mapping $T: C \rightarrow C$ is said to be $\alpha$-demicontractive [17] if $F(T) \neq \emptyset$ and there exist $\lambda>0, \alpha \geq 1$ such that

$$
\langle x-T x, x-\alpha p\rangle \geq \lambda\|x-T x\|^{2}, \forall x \in C \text { and } \forall p \in F(T)
$$

Clearly (1.6) is equivalent to

$$
\|T x-\alpha p\|^{2} \leq\|x-\alpha p\|^{2}+k\|x-T x\|^{2}, \forall x \in C \text { and } \forall p \in F(T),
$$

where $k=1-2 \lambda \in[0,1)$. It is easy to observe that if $T$ is $\alpha$-demicontractive, then $\alpha p \in F(T) \forall p \in F(T)$ such that $\alpha p$ remains in the domain $D(T)$ of $T$. Since if $T$ is demicontractive, then $F(T)$ is closed and convex, it follows that if $T$ is both demicontractive (1-demicontractive) and $\alpha$-demicontractive, $\alpha>1$ 
then the line segment $(1-t) p+t \alpha p, t \in[0,1]$, is contained in $F(T), \forall p \in F(T)$ such that $\alpha p$ remains in the domain $D(T)$ of $T$. In [17] an example of an $\alpha$-demicontractive mapping with $\alpha>1$ which is not demicontractive is given and it is easy to observe that there are demicontractive (1-demicontractive) maps which are not $\alpha$-demicontractive for $\alpha>1$ (see for example ([4], Example 2.2)). For other properties of this new class of demicontractive mappings, the reader may consult [17].

In [17] the authors proved the following strong convergence theorem:

Theorem 1.2 ([17] Theorem 5). Let $C$ be a closed convex subset of a real Hilbert space $H$ and let let $T: C \rightarrow C$ be a demicontractive mapping with constant $k$, or, equivalently $T$ satisfies condition $(A)$ with $\lambda=\frac{(1-k)}{2}$. Let $T$ be $\alpha$-demicontractive for some $\alpha>1$ and let $(I-T)$ be demiclosed at 0 . Let $\left\{\alpha_{n}\right\}_{n=1}^{\infty}$ be a real sequence in $[0,1]$ which satisfy the condition $0<a \leq \alpha_{n} \leq$ $b<2 \lambda=1-k$. Then for suitable $x_{0} \in C$, the sequence $\left\{x_{n}\right\}_{n=1}^{\infty}$ of the Mann iteration sequence given by (1.3) converges strongly to a fixed point of $T$.

It is our purpose in this paper to study the more general class of $\alpha$ demicontractive mappings for which $k=1$ and which we call $\alpha$-hemicontractive mappings following the usual terminology. For this more general class of mapppings, we prove strong convergence theorem similar to Theorem 1.2 using the Ishikawa iteration scheme.

\section{Main Results}

Definition 2.1. We say that a mapping $T: C \rightarrow C$ is $\alpha$-hemicontractive if $F(T) \neq \emptyset$ and there exists $\alpha \geq 1$ such that

$$
\|T x-\alpha p\|^{2} \leq\|x-\alpha p\|^{2}+\|x-T x\|^{2}, \forall x \in C \text { and } \forall p \in F(T) .
$$

Observe that (2.1) is equivalent to

$$
\langle x-T x, x-\alpha p\rangle \geq 0, \forall x \in C \text { and } \forall p \in F(T) .
$$

We discuss the following examples.

Example 2.1. ([4], Example 2.4) Let $\Re$ denote the reals with the usual norm and let $C=[0,1]$. Define $T: C \rightarrow C$ by

$$
T x=\left\{\begin{array}{l}
\frac{1}{2}, x \in\left[0, \frac{1}{2}\right], \\
0, x \in\left(\frac{1}{2}, 1\right] .
\end{array}\right.
$$


Then $T$ is hemicontractive (1-hemicontractive) but not $\alpha$-hemicontractive for some $\alpha>1$. $T$ is neither demicontractive (1-demicontractive) nor $\alpha$ demicontractive for some $\alpha>1$.

Example 2.2. Let $\Re$ denote the reals with the usual norm and let $C=$ $[1,4] \subset \Re$. Define $T: C \rightarrow C$ by;

$$
T x=\left\{\begin{array}{l}
x^{2}, \quad 1 \leq x \leq 2 \\
1,2<x \leq 4
\end{array}\right.
$$

Then $T$ is 2 -hemicontractive (i.e., $T$ is $\alpha$-hemicontractive with $\alpha=2$ ). $T$ is not hemicontractive (1-hemicontractive).

Example 2.3. ([21]) Let $\Re$ denote the reals with the usual norm and let $C=(-\infty, 1)$. Define $T: C \rightarrow C$ by

$$
T x= \begin{cases}\frac{x}{1-x}, & x \in(-\infty, 0] \\ \frac{x}{x-1}, & x \in[0,1)\end{cases}
$$

Then $T$ is hemicontractive (1-hemicontractive) and is also $\alpha$-hemicontractive for all $\alpha>1$. $T$ is neither demicontractive (1-demicontractive) nor $\alpha$ demicontractive for some $\alpha>1$.

Remark 2.1. It is easy to verify that if $T$ is hemicontractive and

$$
\langle x-T x, p\rangle \leq 0, \forall(x, p) \in C \times F(T),
$$

then $T$ is $\alpha$-hemicontractive for all $\alpha>1$.

In [13] the authors proved the following:

Theorem 2.1. ([13], Theorem 1.) Let $H$ be a real Hilbert space and $C$ a nonempty closed convex subset of $H$. Let $T: C \rightarrow C$ be a Lipschitz hemicontractive mapping. Let $\left\{a_{n}\right\},\left\{b_{n}\right\},\left\{c_{n}\right\},\left\{a_{n}^{\prime}\right\},\left\{b_{n}^{\prime}\right\}$ and $\left\{c_{n}^{\prime}\right\}$ be real sequences in $[0,1]$ satisfying the conditions:

(i) $a_{n}+b_{n}+c_{n}=a_{n}^{\prime}+b_{n}^{\prime}+c_{n}^{\prime}=1, n \geq 1$,

(ii) $0<\epsilon \leq b_{n}^{\prime} \leq b_{n} \leq b<1, \forall n \geq 1$, for some $\epsilon>0$ and for some $b \in$ $\left(0, \frac{1}{\left[\left(\sqrt{1+L^{2}}\right)+1\right]}\right)$,

(iii) $\sum_{n=1}^{\infty} c_{n}<\infty, \sum_{n=1}^{\infty} c_{n}^{\prime}<\infty$.

Let $\left\{u_{n}\right\}$ and $\left\{v_{n}\right\}$ be bounded sequences in $C$ and let $\left\{x_{n}\right\}$ be the sequence generated from an arbitrary $x_{1} \in C$ by

$$
x_{n+1}=a_{n}^{\prime} x_{n}+b_{n}^{\prime} T\left[a_{n} x_{n}+b_{n} T x_{n}+c_{n} u_{n}\right]+c_{n}^{\prime} v_{n}, n \geq 1 .
$$

Then $\lim _{n \rightarrow \infty}\left\|x_{n}-T x_{n}\right\|=0$. 
Remark 2.2. If in Theorem $2.1(I-T)$ is demiclosed at 0 , then $\left\{x_{n}\right\}$ converges weakly to a fixed point of $T$ (see for example Theorem 2 of [13]).

As in the case of demicontractive maps, hemicontractiveness and the demiclosedness principle are not sufficient to obtain strong convergence of the Ishikawa scheme to a fixed point of $T$. Additional conditions are required on the map and or the subset $C$. In [22] the authors assumed that the interior of $F(T)$ is nonempty $(\operatorname{int}(F(T)) \neq \emptyset)$ to achieve strong convergence. This appears very restrictive since even in $\Re$ with the usual norm, Lipschitz hemicontractive maps with finite number of fixed points do not enjoy this condition that $\operatorname{int}(F(T)) \neq \emptyset$.

Remark 2.3. The assumption that $T$ is demicontractive (1 - demicontractive) in Theorem 1.2 is to ensure the weak convergence of $\left\{x_{n}\right\}$ to a point $p \in F(T)$. This assumption appears unnecessary since the following argument shows that weak convergence of $\left\{x_{n}\right\}$ to a point $p \in F(T)$ is guaranteed if $T$ is $\alpha$-demicontractive for some $\alpha>1$. If $T: C \rightarrow C$ is $\alpha$-demicontractive with some $\alpha>1$, let $p \in F(T)$ be arbitrary. Then using the well known identity

$$
\|(1-t) x+t y\|^{2}=(1-t)\|x\|^{2}+t\|y\|^{2}-t(1-t)\|x-y\|^{2}
$$

which holds for all $x, y$ in $H$ and for all $t$ in $[0,1]$ we obtain

$$
\begin{aligned}
\left\|x_{n+1}-\alpha p\right\|^{2}= & \left\|\left(1-\alpha_{n}\right)\left(x_{n}-\alpha p\right)+\alpha_{n}\left(T x_{n}-\alpha p\right)\right\|^{2} \\
= & \left(1-\alpha_{n}\right)\left\|x_{n}-\alpha p\right\|^{2}+\alpha_{n}\left\|T x_{n}-\alpha p\right\|^{2} \\
& -\alpha_{n}\left(1-\alpha_{n}\right)\left\|x_{n}-T x_{n}\right\|^{2} \\
\leq & \left\|x_{n}-\alpha p\right\|^{2}-\alpha_{n}\left[1-\alpha_{n}-k\right]\left\|x_{n}-T x_{n}\right\|^{2} \\
\leq & \left\|x_{n}-\alpha p\right\|^{2}-a[1-k-b]\left\|x_{n}-T x_{n}\right\|^{2} .
\end{aligned}
$$

It follows from (2.5) that $\lim _{n \rightarrow \infty}\left\|x_{n}-\alpha p\right\|$ exists for all $p \in F(T)$, and $\lim _{n \rightarrow \infty}\left\|x_{n}-T x_{n}\right\|=0$. Since $\left\{x_{n}\right\}$ is bounded, it has a subsequence say $\left\{u_{n}\right\}_{n=1}^{\infty}$ which converges weakly to a point $u \in C$. Since $\lim _{n \rightarrow \infty}\left\|u_{n}-T u_{n}\right\|=0$ and $(I-T)$ is demiclosed at 0 , then $u \in F(T)$. To conclude that $\left\{x_{n}\right\}$ converges weakly to $u$, it suffices to show that if $\left\{x_{n}\right\}$ has any other subsequence $\left\{v_{n}\right\}_{n=1}^{\infty}$ which converges weakly to $v$, then $u=v$. Observe that we also have that $v \in F(T)$ and thus $\lim _{n \rightarrow \infty}\left\|x_{n}-\alpha u\right\|$ and $\lim _{n \rightarrow \infty}\left\|x_{n}-\alpha v\right\|$ exist. Let $\lim _{n \rightarrow \infty}\left\|x_{n}-\alpha u\right\|=d_{1}$ and $\lim _{n \rightarrow \infty}\left\|x_{n}-\alpha v\right\|=d_{2}$ and consider the sequence

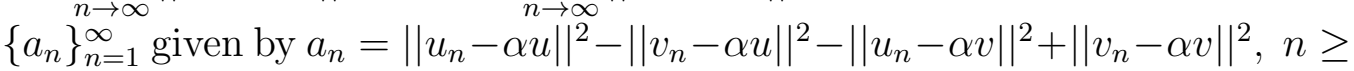
1. Observe that $\lim _{n \rightarrow \infty} a_{n}=0$. Observe also that $a_{n}=-2 \alpha\left\langle u_{n}-v_{n}, u-v\right\rangle$ and 
the weak convergence of $\left\{u_{n}\right\}$ and $\left\{v_{n}\right\}$ to $u$ and $v$ respectively imply that $\lim _{n \rightarrow \infty} a_{n}=-\alpha\|u-v\|^{2}$. Hence $-\alpha\|u-v\|^{2}=0$ and $u=v$.

We now prove the following.

Theorem 2.2. Let $C$ be a nonempty closed convex subset of a real Hilbert space $H$ and let $T: C \rightarrow C$ be an L-Lipschitzian and $\alpha$-hemicontractive mapping with $\alpha>1$. Let $\left\{\alpha_{n}\right\}$ and $\left\{\beta_{n}\right\}$ be real sequences in $[0,1]$ which satisfy the condition $0<\epsilon \leq \alpha_{n} \leq \beta_{n} \leq b<1$ for some $\epsilon>0$ and for some $b \in\left(0, \frac{1}{\sqrt{1+L^{2}}+1}\right)$. Let $(I-T)$ be demiclosed at 0 . Then for suitable $x_{1} \in K$, the sequence $\left\{x_{n}\right\}$ given by

$$
x_{n+1}=\left(1-\alpha_{n}\right) x_{n}+\alpha_{n} T\left[\left(1-\beta_{n}\right) x_{n}+\beta_{n} T x_{n}\right], n \geq 1
$$

converges strongly to a point in $F(T)$.

Proof. Let $G_{n} x_{n}:=T\left[\left(1-\beta_{n}\right) x_{n}+\beta_{n} T x_{n}\right], n \geq 1$. Then for all $p \in F(T)$ we have

$$
\begin{aligned}
\left\|G_{n} x_{n}-\alpha p\right\|^{2}= & \left\|T\left[\left(1-\beta_{n}\right) x_{n}+\beta_{n} T x_{n}\right]-\alpha p\right\|^{2} \\
\leq & \left\|\left(1-\beta_{n}\right) x_{n}+\beta_{n} T x_{n}-\alpha p\right\|^{2} \\
& +\left\|\left(1-\beta_{n}\right) x_{n}+\beta_{n} T x_{n}-G_{n} x_{n}\right\|^{2} \\
& \\
= & \left\|\left(1-\beta_{n}\right)\left(x_{n}-\alpha p\right)+\beta_{n}\left(T x_{n}-\alpha p\right)\right\|^{2} \\
& +\|\left(1-\beta_{n}\right)\left(x_{n}-G_{n} x_{n}\right) \\
& +\beta_{n}\left(T x_{n}-T\left[\left(1-\beta_{n}\right) x_{n}+\beta_{n} T x_{n}\right]\right) \|^{2} \\
= & \left(1-\beta_{n}\right)\left\|x_{n}-\alpha p\right\|^{2}+\beta_{n}\left\|T x_{n}-\alpha p\right\|^{2} \\
& -\beta_{n}\left(1-\beta_{n}\right)\left\|x_{n}-T x_{n}\right\|^{2}+\left(1-\beta_{n}\right)\left\|x_{n}-G_{n} x_{n}\right\|^{2} \\
& +\beta_{n}\left\|T x_{n}-T\left[\left(1-\beta_{n}\right) x_{n}+\beta_{n} T x_{n}\right]\right\|^{2} \\
& -\beta_{n}\left(1-\beta_{n}\right)\left\|x_{n}-T x_{n}\right\|^{2} \\
\leq & \left\|x_{n}-\alpha p\right\|^{2}+\beta_{n}\left\|x_{n}-T x_{n}\right\|^{2}-\beta_{n}\left(1-\beta_{n}\right)\left\|x_{n}-T x_{n}\right\|^{2} \\
& +\left(1-\beta_{n}\right)\left\|x_{n}-G_{n} x_{n}\right\|^{2}+L^{2} \beta_{n}^{3}\left\|x_{n}-T x_{n}\right\|^{2} \\
& -\beta_{n}\left(1-\beta_{n}\right)\left\|x_{n}-T x_{n}\right\|^{2} \\
= & \left\|x_{n}-\alpha p\right\|^{2}+\left(1-\beta_{n}\right)\left\|x_{n}-G_{n} x_{n}\right\|^{2} \\
& -\beta_{n}\left[1-2 \beta_{n}-\beta_{n}^{2} L^{2}\right]\left\|x_{n}-T x_{n}\right\|^{2} .
\end{aligned}
$$

Using the condition on $\left\{\beta_{n}\right\}$ in (2.7) we obtain

$$
\left\|G_{n} x_{n}-\alpha p\right\|^{2} \leq\left\|x_{n}-\alpha p\right\|^{2}+\left(1-\beta_{n}\right)\left\|x_{n}-G_{n} x_{n}\right\|^{2} .
$$


It follows easily from (2.8) that

$$
\left\langle x_{n}-G_{n} x_{n}, x_{n}-\alpha p\right\rangle \geq \frac{\beta_{n}}{2}\left\|x_{n}-G_{n} x_{n}\right\|^{2} .
$$

Using (2.7) we obtain for arbitrary $p \in F(T)$ that

$$
\begin{aligned}
& \left\|x_{n+1}-\alpha p\right\|^{2}=\left\|\left(1-\alpha_{n}\right)\left(x_{n}-\alpha p\right)+\alpha_{n}\left(G_{n} x_{n}-\alpha p\right)\right\|^{2} \\
& =\left(1-\alpha_{n}\right)\left\|x_{n}-\alpha p\right\|^{2}+\alpha_{n}\left\|G_{n} x_{n}-\alpha p\right\|^{2} \\
& -\alpha_{n}\left(1-\alpha_{n}\right)\left\|x_{n}-G_{n} x_{n}\right\|^{2} \\
& \leq\left(1-\alpha_{n}\right)\left\|x_{n}-\alpha p\right\|^{2}+\alpha_{n}\left[\left\|x_{n}-\alpha p\right\|^{2}\right. \\
& +\left(1-\beta_{n}\right)\left\|x_{n}-G_{n} x_{n}\right\|^{2} \\
& \left.-\beta_{n}\left(1-2 \beta_{n}-\beta_{n}^{2} L^{2}\right)\left\|x_{n}-T x_{n}\right\|^{2}\right] \\
& -\alpha_{n}\left(1-\alpha_{n}\right)\left\|x_{n}-G_{n} x_{n}\right\|^{2} \\
& =\left\|x_{n}-\alpha p\right\|^{2}-\alpha_{n}\left(\beta_{n}-\alpha_{n}\right)\left\|x_{n}-G_{n} x_{n}\right\|^{2} \\
& -\alpha_{n} \beta_{n}\left[1-2 \beta_{n}-\beta_{n}^{2} L^{2}\right]\left\|x_{n}-T x_{n}\right\|^{2} \\
& \leq\left\|x_{n}-\alpha p\right\|^{2}-\alpha_{n} \beta_{n}\left[1-2 \beta_{n}-\beta_{n}^{2} L^{2}\right]\left\|x_{n}-T x_{n}\right\|^{2} \\
& \leq\left\|x_{n}-\alpha p\right\|^{2}-\epsilon^{2}\left[1-2 b-b^{2} L^{2}\right]\left\|x_{n}-T x_{n}\right\|^{2} \text {. }
\end{aligned}
$$

It follows from (2.10) that $\lim _{n \rightarrow \infty}\left\|x_{n}-\alpha p\right\|$ exists for all $p \in F(T)$, and $\lim _{n \rightarrow \infty}\left\|x_{n}-T x_{n}\right\|=0$.

Thus as in Remark 2.3 we obtain that $\left\{x_{n}\right\}$ converges weakly to some $p \in F(T)$. We prove that

$$
\left\langle x_{n}-p, p\right\rangle \geq \frac{1}{2(\alpha-1)}\left\|x_{n}-p\right\|^{2}, \forall n \geq 1 .
$$

We choose $x_{1} \in C$ (see for example [17]) such that

$$
\left\langle x_{1}-p, p\right\rangle \geq \frac{1}{2(\alpha-1)}\left\|x_{1}-p\right\|^{2} .
$$

Suitable $x_{1} \in C$ exists since if $P_{C}: H \rightarrow C$ is the proximity map (projection map from $H$ onto $C$ ), then for $\lambda \in \Re$ such that $1<\lambda \leq 2 \alpha-1$, we can choose $x_{1}=P_{C}(\lambda p)$.

Then since the proximity map, $P_{C}$ is firmly nonexpansive (i.e., $\| P_{C} x-$ $\left.P_{C} y \|^{2} \leq\left\langle P_{C} x-P_{C} y, x-y\right\rangle\right)$, it is easy to verify that $x_{1}$ satisfies $(2.12)$. 
The proof of (2.11) now follows by induction since if we assume (2.11), then from (2.9) and $\alpha>1$ we obtain

$$
\begin{aligned}
& \left\langle x_{n}-G_{n} x_{n}, x_{n}-\alpha p\right\rangle \geq \frac{\beta_{n}}{2}\left\|x_{n}-G_{n} x_{n}\right\|^{2} \geq \frac{\alpha_{n}}{2}\left\|x_{n}-G_{n} x_{n}\right\|^{2} \\
\Rightarrow \quad & \left\langle x_{n}-G_{n} x_{n}, x_{n}-p-(\alpha-1) p\right\rangle \geq \frac{\alpha_{n}}{2}\left\|x_{n}-G_{n} x_{n}\right\|^{2} \\
\Rightarrow \quad & -(\alpha-1)\left\langle x_{n}-G_{n} x_{n}, p\right\rangle \geq-\left\langle x_{n}-G_{n} x_{n}, x_{n}-p\right\rangle \\
& +\frac{\alpha_{n}}{2}\left\|x_{n}-G_{n} x_{n}\right\|^{2} \\
\Rightarrow \quad & -\left\langle x_{n}-G_{n} x_{n}, p\right\rangle \geq-\frac{1}{(\alpha-1)}\left\langle x_{n}-G_{n} x_{n}, x_{n}-p\right\rangle \\
& +\frac{\alpha_{n}}{2(\alpha-1)}\left\|x_{n}-G_{n} x_{n}\right\|^{2} \\
& \quad-\alpha_{n}\left\langle x_{n}-G_{n} x_{n}, p\right\rangle \geq-\frac{\alpha_{n}}{(\alpha-1)}\left\langle x_{n}-G_{n} x_{n}, x_{n}-p\right\rangle \\
& +\frac{\alpha_{n}^{2}}{2(\alpha-1)}\left\|x_{n}-G_{n} x_{n}\right\|^{2},
\end{aligned}
$$

and with the inductive hypothesis (2.11) we obtain

$$
\begin{aligned}
\left\langle x_{n}-p-\alpha_{n}\left(x_{n}-G_{n} x_{n}\right), p\right\rangle \geq & \frac{1}{2(\alpha-1)}\left\|x_{n}-p\right\|^{2} \\
& -\frac{\alpha_{n}}{(\alpha-1)}\left\langle x_{n}-G_{n} x_{n}, x_{n}-p\right\rangle \\
& +\frac{\alpha_{n}^{2}}{2(\alpha-1)}\left\|x_{n}-G_{n} x_{n}\right\|^{2}, \\
\Rightarrow\left\langle\left(1-\alpha_{n}\right) x_{n}+\alpha_{n} G_{n} x_{n}-p, p\right\rangle \geq & \frac{1}{2(\alpha-1)}\left[\left\|x_{n}-p\right\|^{2}\right. \\
& -2 \alpha_{n}\left\langle x_{n}-G_{n} x_{n}, x_{n}-p\right\rangle \\
& \left.+\alpha_{n}^{2}\left\|x_{n}-G_{n} x_{n}\right\|^{2}\right] \\
\Rightarrow\left\langle\left(1-\alpha_{n}\right) x_{n}+\alpha_{n} G_{n} x_{n}-p, p\right\rangle \geq & \frac{1}{2(\alpha-1)}\left\|x_{n}-p-\alpha_{n}\left(x_{n}-G_{n} x_{n}\right)\right\|^{2} \\
\Rightarrow\left\langle x_{n+1}-p, p\right\rangle \geq \frac{1}{2(\alpha-1)} \| x_{n+1} & -p \|^{2} .
\end{aligned}
$$

Since $\left\{x_{n}\right\}$ converges weakly to $p$, we have that $\lim _{n \rightarrow \infty}\left\|x_{n}-p\right\|=0$.

Remark 2.4. If $C$ is a nonempty closed convex subset of a real Hilbert space $H$ and $T: C \rightarrow C$ is an $L$-Lipschizian $\alpha$-hemicontractive map, $\alpha \geq 1$, then 
$\alpha p \in F(T)$ for all $p \in F(T)$ such that $\alpha p$ remains in the domain $D(T)$ of $T$. Furthermore, since if $T$ is $L$-Lipschtzian hemicontractive, then $F(T)$ is closed and convex, it follows that if $T$ is both $L$-Lipschitzian hemictractive and $\alpha$ hemicontractive, $\alpha>1$, then the line segment $(1-t) p+t(\alpha p), t \in[0,1]$, is contained in $F(T)$ for all $p \in F(T)$ such that $\alpha p$ remains in the domain $D(T)$ of $T$.

\section{Acknowledgement}

The work was started when the first author was visiting the Abdus Salam International Centre for Theoretical Physics (ICTP), Trieste, Italy as an Associate. He is grateful to the Centre for the invaluable facilities at the Centre and for hospitality. The authors are grateful to the referee for his useful comments and suggestions.

\section{References}

[1] T.L. Hicks and J.D. Kubicek, On the Mann iteration process in a Hilbert spaces, J. Math. Anal. Appl., 59, (1977), 498-504

[2] Ş. Măruşter, Sur le calcul des zeros d'un operateur discontinu par iteration, Canad. Math. Bull., 16 (4), (1973), 541-544

[3] Ş. Măruşter, The solution by iteration of nonlinear equations in Hilbert spaces, Proc. Amer. Math Soc., 63 (1), (1977), 69-73

[4] S.A. Naimpally and K.L. Singh, Extensions of some fixed point theorems of Rhoades, J. Math. Anal. Appl., 96, (1983), 437-446

[5] C.E. Chidume and S. Măruşter, Iterative methods for the computation of fixed points of demicontractive mappings, J. Comput. Appl. Math., 234 (3), (2010), 861882.

[6] D. Boonchari and S. Saejung, Construction of common fixed points of a countable family of $\lambda$-demicontractive mappings in arbitrary Banach spaces, Appl. Math. Comput., 216, (2010), 173-178.

[7] P.E. Maingé, Regularized and inertial algorithms for common fixed points of nonlinear operators, J. Math. Anal. Appl., 344, (2008), 876-887.

[8] M.O. Osilike, Strong and weak convergence of the Ishikawa iteration method for a class of nonlinear equations, Bull. Korean Math. Soc., 37 (1), (2000), 153-169.

[9] M.O. Osilike, Iterative approximation of fixed points of asymptotically demicontractive maps, Indian J. Pure Appl. Math., 29 (12), (1998), 1291-1300. 
[10] P.E. Maingé and Ş. Măruşter, Convergence in norm of modified KrasnoselskiMann iterations for fixed points of demicontractive mappings, Appl. Math. Comput., 217 (24), (2011), 9864-9874.

[11] C.E. Chidume, The solution by iteration of nonlinear equations in certain Banach spaces, J. Nigerian Math. Soc., 3, (1984), 57-62.

[12] A.M. Saddeek, Iterative method for nonlinear pseudo-monotone type operators in Hilbert spaces, Far East J. Appl. Math., 32 (1), (2008), 33-40.

[13] M.O. Osilike and D.I. Igbokwe, Weak and Strong Convergence Theorems for Fixed Points of Pseudocontractions and Solutions of Monotone Type Operator Equations, Comput. Math. Appl., 40, (2000), 559-567.

[14] X. Weng, The iterative solution of nonlinear equations in certain Banach spaces, $J$. Nigerian Math. Soc., 11, (1992), 1-7.

[15] C.E. Chidume and S.A. Mutangadura, An example on the Mann iteration method for Lipschitz pseudocontractions, Proc. Amer. Math. Soc., 129, (2001), 23592363.

[16] A. Genel and J. Lindenstraus, An example concerning fixed points, Israel $J$. Math., 22 (1), (1975), 81-86.

[17] L. Măruşter and Ş. Măruşter, Strong convergence of the Mann iteration for $\alpha$ demicontractive mappings, Math. Comput. Model., 54, (2011), 2486-2492.

[18] K. Nakajo and W. Takahashi, Strong convergence theorems for nonexpansive mappings and nonexpansive semigroups, J. Math. Anal. Appl., 279, (2003), 372-379.

[19] L. Ciric, A. Rafiq, S. Radenovic, M. Racovic and S.U. Ume, On the Mann implicit iteration for strongly accretive and strongly pseudo-contractive mappings, Appl. Math. Comput., 198, (2008), 128-137.

[20] Rafiq A., On Mann iteration in Hilbert spaces, Nonlinear Anal., 66, (2007), 22302236 .

[21] M.O. Osilike and F.O. Isiogugu, Fixed points of asymptotically $\phi$-demicontractive maps in arbitrary Banach spaces, 15 (3), (2005), 59-67.

[22] H. Zegeye, N. Shahzad and M.A. Alghamdi, Convergence of Ishikawas iteration method for pseudocontractive mappings, Nonlinear Anal., 74, (2011), 7304-7311.

Micah Okwuchukwu Osilike

Department of Mathematics, University of Nigeria, Nsukka, Nigeria

E-mail: osilike@yahoo.com, micah.osilike@unn.edu.ng

Anthony Chibuike Onah

Department of Mathematics, University of Nigeria, Nsukka, Nigeria

E-mail: onah87@gmail.com

Received: 23.02.2015

Accepted: 17.03.2015 